\title{
Cholinergic Neurons in the Basal Forebrain Promote Wakefulness by Actions on Neighboring Non-Cholinergic Neurons: An Opto-Dialysis Study
}

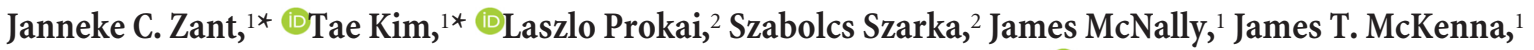 \\ Charu Shukla, ${ }^{1}$ Chun Yang, ${ }^{1}$ Anna V. Kalinchuk, ${ }^{1}$ Robert W. McCarley, ${ }^{1}{ }^{\circ}$ Ritchie E. Brown, ${ }^{1}$ and Radhika Basheer ${ }^{1}$ \\ ${ }^{1}$ Veterans Administration Boston Healthcare System and Harvard Medical School Department of Psychiatry, West Roxbury, Massachusetts 02132 , and \\ ${ }^{2}$ Center for Neuroscience Discovery, Institute for Healthy Aging, University of North Texas Health Science Center, Fort Worth, Texas 76107
}

Understanding the control of sleep-wake states by the basal forebrain $(\mathrm{BF})$ poses a challenge due to the intermingled presence of cholinergic, GABAergic, and glutamatergic neurons. All three BF neuronal subtypes project to the cortex and are implicated in cortical arousal and sleep-wake control. Thus, nonspecific stimulation or inhibition studies do not reveal the roles of these different neuronal types. Recent studies using optogenetics have shown that "selective" stimulation of BF cholinergic neurons increases transitions between NREM sleep and wakefulness, implicating cholinergic projections to cortex in wake promotion. However, the interpretation of these optogenetic experiments is complicated by interactions that may occur within the BF. For instance, a recent in vitro study from our group found that cholinergic neurons strongly excite neighboring GABAergic neurons, including the subset of cortically projecting neurons, which contain the calcium-binding protein, parvalbumin (PV) (Yang et al., 2014). Thus, the wake-promoting effect of "selective" optogenetic stimulation of $\mathrm{BF}$ cholinergic neurons could be mediated by local excitation of GABA/PV or other non-cholinergic BF neurons. In this study, using a newly designed opto-dialysis probe to couple selective optical stimulation with simultaneous in vivo microdialysis, we demonstrated that optical stimulation of cholinergic neurons locally increased acetylcholine levels and increased wakefulness in mice. Surprisingly, the enhanced wakefulness caused by cholinergic stimulation was abolished by simultaneous reverse microdialysis of cholinergic receptor antagonists into BF. Thus, our data suggest that the wake-promoting effect of cholinergic stimulation requires local release of acetylcholine in the basal forebrain and activation of cortically projecting, non-cholinergic neurons, including the GABAergic/PV neurons.

Key words: basal forebrain; cholinergic neurons; NREM to wake transitions; opto-dialysis

Significance Statement

Optogenetics is a revolutionary tool to assess the roles of particular groups of neurons in behavioral functions, such as control of sleep and wakefulness. However, the interpretation of optogenetic experiments requires knowledge of the effects of stimulation on local neurotransmitter levels and effects on neighboring neurons. Here, using a novel "opto-dialysis" probe to couple optogenetics and in vivo microdialysis, we report that optical stimulation of basal forebrain (BF) cholinergic neurons in mice increases local acetylcholine levels and wakefulness. Reverse microdialysis of cholinergic antagonists within BF prevents the wake-promoting effect. This important result challenges the prevailing dictum that BF cholinergic projections to cortex directly control wakefulness and illustrates the utility of "opto-dialysis" for dissecting the complex brain circuitry underlying behavior.

\section{Introduction}

The basal forebrain (BF) cholinergic neurons, with their widespread projections to neocortical and paralimbic regions of the

Received Sept. 3, 2015; revised Dec. 7, 2015; accepted Dec. 23, 2015.

Author contributions: J.C.Z., T.K., L.P., C.Y., R.E.B., and R.B. designed research; J.C.Z., T.K., L.P., S.S., J.M., J.T.M., C.S., C.Y., A.V.K., and R.B. performed research; R.B. contributed unpublished reagents/analytic tools; J.C.Z., T.K., L.P., S.S., J.M., J.T.M., C.S., C.Y., A.V.K., R.E.B., and R.B. analyzed data; J.C.Z., T.K., L.P., R.W.M., R.E.B., and R.B. wrote the paper. brain, were the first BF neurons to be recognized for their role in cortical EEG desynchronization and arousal (Metherate et al., 1992; Szymusiak, 1995; Sarter and Bruno, 2000; Zaborszky and 
Duque, 2003; Jones, 2004; Brown et al., 2012). Indeed, many neuroscientists currently equate BF behavioral state control with the cortical projections of cholinergic neurons. However, recent studies have implicated other cortically projecting BF neurons releasing GABA or glutamate in control of cortical EEG and sleep-wake states (Duque et al., 2000; Manns et al., 2003; Anaclet et al., 2015; Kim et al., 2015; Lin et al., 2015; Xu et al., 2015). Thus, interactions of cholinergic neurons with non-cholinergic BF neurons may be important in orchestrating arousal.

In the past decade, optogenetics has provided a revolutionary approach toward establishing causal relationships between specific neuronal circuits and behavior (Deisseroth, 2015). In particular, optogenetics has been useful in dissecting out the role of specific neurotransmitter systems embedded in brain regions with heterogeneous neuronal phenotypes (Adamantidis et al., 2007; Han et al., 2014; Irmak and de Lecea, 2014; Kim et al., 2015; $\mathrm{Xu}$ et al., 2015). However, the attribution of effects of optogenetic stimulation on behavior to activation of particular neurotransmitter systems and release of that neurotransmitter in target areas is not as simple as it first seems. Many neuromodulatory systems with long projecting axons also release neurotransmitter(s) locally through somatodendritic release (Adell and Artigas, 2004) or through activation of local axon collaterals (Duque et al., 2007; Yang et al., 2014). Thus, the behavioral effects of "selective" optogenetic stimulation may be mediated not through release of neurotransmitter in distal targets but rather due to activation or inhibition of neighboring neurons.

Recent optogenetic studies reported that selective activation of BF cholinergic neurons suppresses slow-wave sleep and promotes wakefulness (Han et al., 2014; Irmak and de Lecea, 2014; $\mathrm{Xu}$ et al., 2015). Furthermore, inhibition of cholinergic neurons prolongs slow-wave sleep and decreases the probability of awakening (Shi et al., 2015). However, these studies did not evaluate the local neurochemical changes associated with optical stimulation, or investigate the influence of cholinergic stimulation on neighboring neurons in vivo. Acetylcholine (ACh) levels in BF are higher during wakefulness and rapid eye movement (REM) sleep compared with non-REM (NREM) sleep (Vazquez and Baghdoyan, 2001), suggesting that modulation of neighboring non-cholinergic neurons may be important in behavioral state control. Consistent with this idea, a recent in vitro study from our group (Yang et al., 2014) found that cholinergic neurons strongly excite neighboring GABAergic neurons, including the subset of cortically projecting neurons, which contain the calcium-binding protein, parvalbumin (PV). In vivo optogenetic and pharmacogenetic studies showed that stimulation of BF GABAergic, PV or glutamatergic neurons promotes cortical activation and wakefulness (Anaclet et al., 2015; Kim et al., 2015; Xu et al., 2015). Moreover, lesions of BF cholinergic neurons did not impair the ability of BF PV neurons to produce cortical activation (Kim et al., 2015). Together, these results suggest that non-cholinergic neu-

LeChasseur of Doric Lenses (Quebec City, Quebec, Canada) for constructing the opto-dialysis probe according to our proposed design; Drs. Robert E. Strecker and Stephen Thankachan for helpful discussions; Varun Konanki and Zeineb Al-Itejawi for EEG scoring; Farzana Pervin for technical assistance; and Diane Ghera, Dewayne Williams, and Alexander Boczanowski for help with animal care.

The authors declare no competing financial interests.

*J.C.Z. and T.K. contributed equally to this work.

Correspondence should be addressed to Dr. Radhika Basheer, Veterans Administration Boston Healthcare System and Harvard Medical School Department of Psychiatry, 1400 VFW Parkway, West Roxbury, MA 02132. E-mail: Radhika_Basheer@hms.harvard.edu.

T. Kim's current address: Department of Psychiatry, Kyung Hee University Hospital at Gangdong, 05278, Seoul, Korea.

DOI:10.1523/JNEUROSCI.3318-15.2016

Copyright $\odot 2016$ the authors $\quad 0270-6474 / 16 / 362058-11 \$ 15.00 / 0$ rons can affect cortical activation and wakefulness independently of cholinergic neurons. These in vitro and in vivo observations highlight an important point that interpretation of optogenetic experiments requires an understanding of the effect of optical stimulation on neurotransmitter release adjacent to the stimulation site, as well as the effects on neighboring, "unstimulated" neurons.

To better understand the circuit mechanisms by which BF cholinergic neurons promote wakefulness, in this study, using a newly designed mouse "opto-dialysis probe" that couples the optical and microdialysis probe in one unit, we measured local changes in ACh levels while performing selective optogenetic stimulation of cholinergic neurons and monitored the effect on sleep-wakefulness. Furthermore, we performed reverse microdialysis of acetylcholine receptor antagonists to block the effect of $\mathrm{ACh}$ within the $\mathrm{BF}$ to determine the importance of its local actions on neighboring non-cholinergic neurons in sleep-wake regulation.

\section{Materials and Methods}

\section{General methods}

Animals. ChAT-ChR2-eYFP mice (strain 014546; The Jackson Laboratory), constitutively expressing channelrhodopsin 2 (ChR2) under the control of the choline acetyltransferase (ChAT) promoter (Zhao et al., 2011), were bred in-house to generate the animals for this study. Mice of either sex ( 8 males and 2 females) were used in our study. Results were similar in males and females and have been pooled. The mice were housed under constant temperature $\left(21 \pm 1^{\circ} \mathrm{C}\right)$ in a $12 \mathrm{~h}$ light/dark cycle (9:00 A.M./9:00 P.M.). Food and water were provided ad libitum.

All experiments conformed to Veterans Administration, Harvard University and National Institutes of Health guidelines on the ethical use of animals. All efforts were made to minimize the number of animals used and their suffering. Experimental procedures were approved by the Institutional Animal Care and Use Committee and Institutional Biosafety Committee of the Veterans Administration Boston Healthcare System.

Opto-dialysis probe design for freely behaving mice. To construct the opto-dialysis probe, we modified a CMA7 microdialysis guide cannula by replacing the polyurethane outer tubing, into which the stylus is inserted, with a stainless steel shaft ( $5 \mathrm{~mm}$ long) to allow a stable attachment of the implantable fiber optic cannula. At the time of the experiment, the stylus was removed and replaced by a microdialysis probe with a 1-mm-long dialysis membrane extending below the guide cannula tip. A fiber optic cannula (core diameter of $200 \mu \mathrm{m}$ ) was glued to the side of the steel guide cannula using a biocompatible material EPO-TEK 302-3M (AngstromBond). As described by Aravanis et al. (2007) and Yizhar et al. (2011), the light scatter emitted from the optical fiber tip decreases exponentially with distance from the fiber tip and only $\sim 1 \%$ of the initial power density reaches a penetration of $0.5 \mathrm{~mm}$ in brain tissue. Therefore, an important consideration in our design was the placement of the microdialysis membrane within the area of the scattered light. To achieve this, the tip of the fiber optic cannula was placed so it extends $0.5 \mathrm{~mm}$ beyond the microdialysis cannula. Thus, when the microdialysis probe is inserted, the 1 $\mathrm{mm}$ dialysis membrane extends $0.5 \mathrm{~mm}$ beyond the tip of the fiber optic cannula, placing the major part of the active zone of the microdialysis membrane within the boundaries of the light scatter (Fig. 1a-c). The fiber optic cannula is connected to a mono fiberoptic patchcord (MFP_200/240/900-0.22_2m_FC-ZF1.25 from Doric Lenses) via a zirconia ferrule and metal flange using a bronze sleeve connector to prevent light leakage at the connection site (Fig. 1a,d). The maximum diameter of the implanted portion of the opto-dialysis probe is $1 \mathrm{~mm}$. The size of the opto-dialysis probe (implanted unilaterally) did not interfere with the implantation of the EEG electrodes (Fig. $1 c, d$ ) and allowed the mouse to move freely in the cage (Fig. 1d). As described below, implantation of the combined opto-dialysis probe allowed both optical stimulation and microdialysis. Post hoc analysis confirmed the location of the probe tips 
a

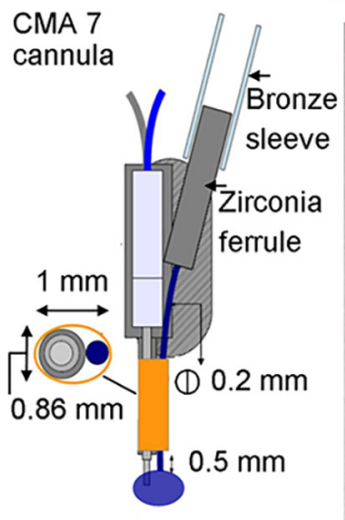

d

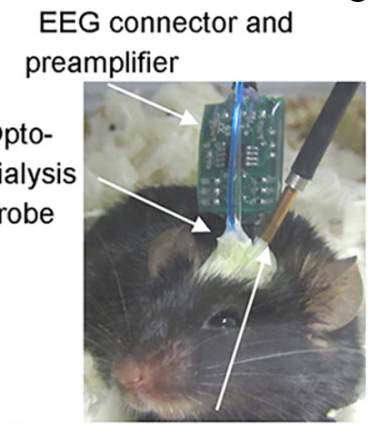

Fiber optic cannula

e b
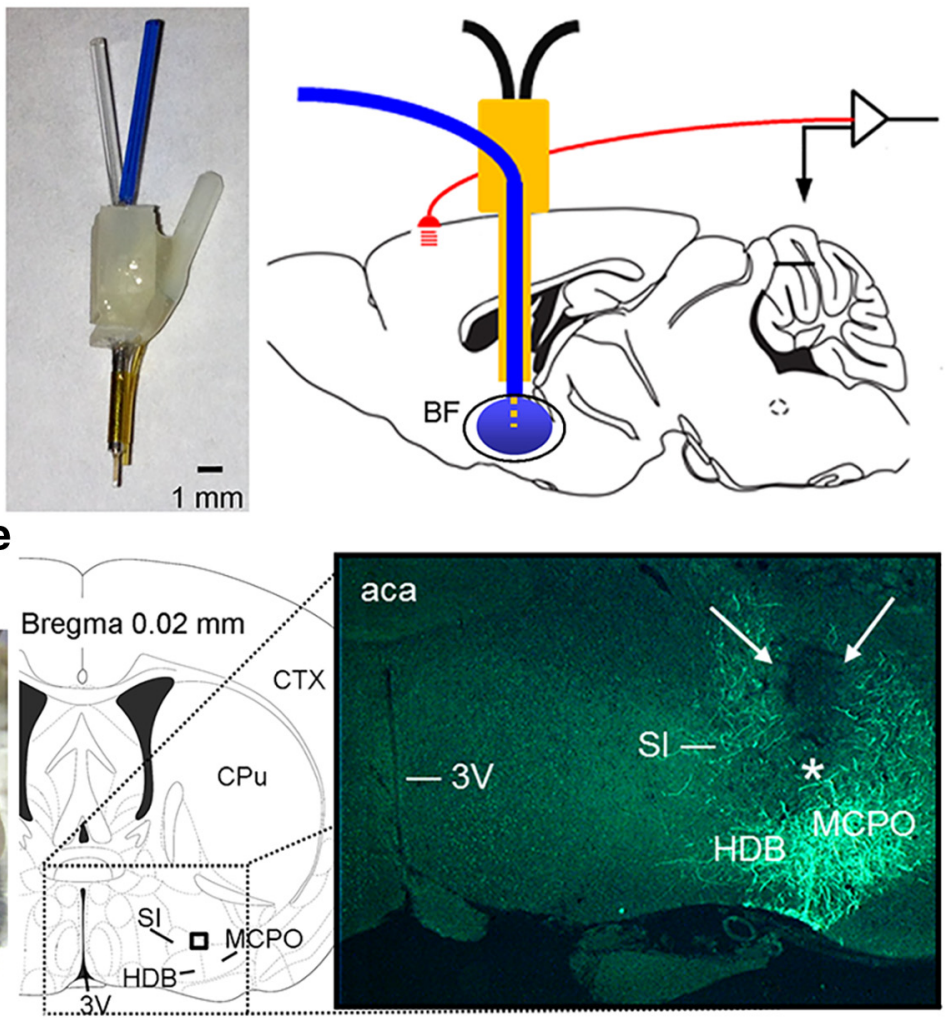

C

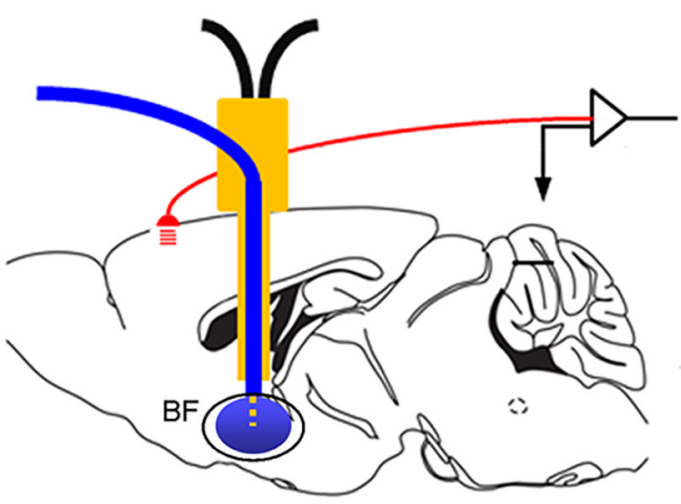

f

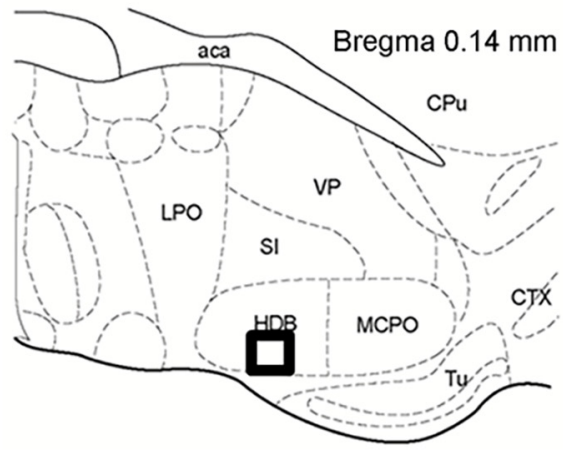

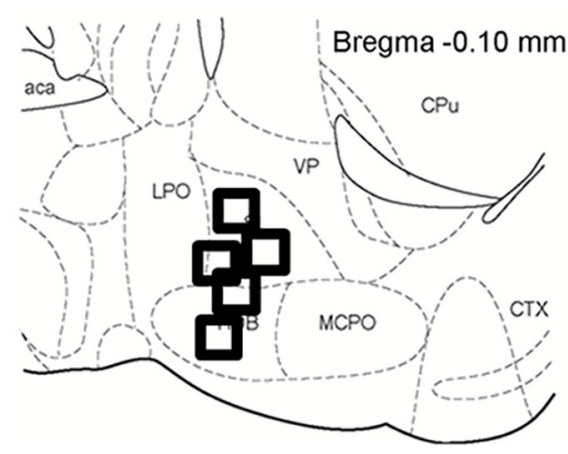

Figure 1. Design of the novel opto-dialysis probe and successful targeting of the BF sleep-wake control center. Diagram (a) and image ( $\boldsymbol{b}$ ) of the opto-dialysis probe. $\boldsymbol{C}$, Sagittal view of the mouse brain illustrates placement of the opto-dialysis probe in the BF and recording of cortical EEG activity. $\boldsymbol{d}$, Image of the opto-dialysis system combined with EEG/EMG in a freely moving mouse. $\boldsymbol{e}$, Representative example of correct targeting of the BF in one mouse. The tip of the microdialysis probe is depicted by a black square in the coronal schematic drawing (left) and by a white asterisk in the image (right). Cholinergic neurons and fibers exhibit green (EYFP) fluorescence. $f$, Center of the probe tip (black squares) within BF for all mice $(n=6)$. All probe tips were located between -0.10 and $0.14 \mathrm{~mm}$ from bregma (Franklin and Paxinos, 2008). 3V, Third ventricle; aca, anterior commissure; CPu, caudate-putamen; CTX, cortex; HDB, horizontal limb of the diagonal band; LP0, lateral preoptic area; MCPO, magnocellular preoptic nucleus; SI, substantia innominata; Tu, olfactory tubercle; VP, ventral pallidum.

within the region of the BF with ChR2-EYFP expressing cholinergic neurons (Fig. 1e,f).

Stereotaxic surgery and opto-dialysis probe implantation. Mice were deeply anesthetized with isoflurane (induction: $3 \%$; maintenance: $1.5 \%-$ $2.5 \%$ ) and placed in a stereotaxic device (Kopf Instruments). After exposing, cleaning, and disinfecting the skull bone, our newly designed opto-dialysis probe (the microdialysis cannula with optic fiber attached to its side) was implanted, targeting the $\mathrm{BF}(\mathrm{AP}=0 ; \mathrm{ML}=1.5$; DV $=-5.6$ ) (Franklin and Paxinos, 2008). Subsequently, two screw electrodes were fitted into the skull for frontal $(\mathrm{AP}=2 ; \mathrm{ML}=1)$ and parietal $(\mathrm{AP}=-3 ; \mathrm{ML}=1.5)$ epidural bipolar recording of the EEG, referenced to a screw-electrode over the cerebellum $(\mathrm{AP}=-5 ; \mathrm{ML}=0)$. To record the electromyogram (EMG), two wire electrodes were inserted into the neck musculature. All electrodes were connected to a prefabricated headmount (catalog \#8402, Pinnacle Technology) and were secured in place with acrylic dental cement. After 1 week of recovery from surgery, the animals were placed in the recording cages in the experiment room. After $2 \mathrm{~d}$ of acclimation, the experiments were started.

Optical stimulation and EEG/EMG recording. Optical stimulation was performed unilaterally to excite cholinergic BF neurons. Laser light (20 $\mathrm{mW}$ ) was generated using a fiber coupled $473 \mathrm{~nm}$ solid-state laser diode (MBL-III-473 nm-50mW, OptoEngine), and was delivered via the optodialysis probe (Doric Lenses). Laser light stimulation was driven by software-generated TTL pulses (WinWCP, Strathclyde Institute of Pharmacy and Biomedical Sciences, Glasgow). Continuous EEG/EMG recordings were performed using Sirenia Acquisition Software in combination with a Pinnacle data acquisition unit (8206 system, Pinnacle Technology). The EEG/EMG signals were amplified (gain 1000), filtered (high pass: $0.5 \mathrm{~Hz}$, low pass $125 \mathrm{~Hz}$ ), and sampled at $400 \mathrm{~Hz}$. EEG was recorded ipsilateral to the opto-dialysis probe.

EEG/EMG analysis. Behavioral states were manually scored in $4 \mathrm{~s} \mathrm{ep-}$ ochs using Sirenia Sleep Pro software (Pinnacle Technology) according 
to standard criteria as follows. Wakefulness: low-amplitude, fast EEG activity with high activity in the EMG; NREM sleep: high-amplitude EEG $\delta(0.5-4 \mathrm{~Hz})$ waves and low-amplitude EMG; REM sleep: regular $\theta(5-9$ $\mathrm{Hz}$ ) activity in the EEG and low or absent EMG activity. Epochs containing artifacts or more than one vigilance state were excluded from further analysis. In all trials that started in NREM sleep, the latency to wakefulness was calculated manually from the start of the stimulation train, indicated by the TTL pulses that were annotated onto the EEG/EMG during the recording. EEG power spectra during wakefulness were calculated within the $0.5-50 \mathrm{~Hz}$ frequency range by fast Fourier transform (256; Hanning window, $0.25 \mathrm{~Hz}$ resolution). Epochs, including the stimulation train itself, were excluded to prevent contamination of the spectra by event-related potential-type responses occurring during the stimulation. Power was expressed as percentage of total power (average of $0.5-20 \mathrm{~Hz}$ during the same experimental day for each individual animal).

In vivo microdialysis. Microdialysis probes (CMA7, membrane length $1 \mathrm{~mm}$, Harvard apparatus/CMA/Microdialysis) were inserted through the guide cannula $22-24 \mathrm{~h}$ before the first experiment. On all experimental days, microdialysis tubing was connected and perfusion of aCSF (Harvard Apparatus) at $1 \mu \mathrm{l} / \mathrm{min}$ was started at Zeitgeber time (ZT) 0.5 and continued until ZT 6; $1 \mathrm{~h}$ samples were collected from ZT 1 onward. EEG/EMG recordings were started at ZT1. The microdialysis probes were left in place throughout the experiment and on nonexperimental days were flushed gently with aCSF. Measurements of neurotransmitter levels were consistent over the course of the experiment, indicating that blockade of the probe was not an issue in our experiments.

Liquid chromatography-tandem mass spectrometry (LC-MS/MS). Microdialysate ACh concentrations were measured using an LC-MS/MS assay developed from earlier procedures (Keski-Rahkonen et al., 2007; Prokai et al., 2008). To $10 \mu \mathrm{l}$ of the collected sample, we added $10 \mu \mathrm{l}$ of aCSF solution containing $200 \mathrm{pg} / \mathrm{ml} \mathrm{ACh}-\mathrm{d}_{4}$ chloride (CDN Isotopes) as an internal standard. After centrifugation at $14,500 \mathrm{rpm}$ for $3 \mathrm{~min}$, the sample was transferred into a $250 \mu$ l plastic autosampler vial that was sealed using an open-top snap cap with a Teflon/soft silicone septum (Thermo Scientific). LC-MS/MS analyses were performed using a Surveyor liquid chromatograph system connected to a TSQ Quantum Ultra triple-quadrupole tandem mass spectrometer (Thermo Scientific). ACh and the internal standard were eluted isocratically at $300 \mu \mathrm{l} / \mathrm{min}$ and $30^{\circ} \mathrm{C}$ from a $100 \mathrm{~mm} \times 2.0 \mathrm{~mm}$ i.d. Synergi Polar-RP column packed with $4 \mu \mathrm{m}$ particles (Phenomenex) with a mobile phase of water/acetonitrile/trifluoroacetic acid mixture (98:2:0.05, v/v). We used a 6 port valve that switched the effluent to enter the ion source between 1.0 and $1.9 \mathrm{~min}$ after the injection of $10 \mu \mathrm{l}$ from the sample by a robotic autosampler of the Surveyor system. The mass spectrometer was operated using atmospheric pressure spray ionization (Keski-Rahkonen et al., 2007), and our method relied on selected-reaction monitoring optimized to the transitions reported previously (Prokai et al., 2008). We prepared calibration samples by serial dilution of $1 \mathrm{ng} / \mathrm{ml}$ ACh chloride (SigmaAldrich) stock solution in aCSF as a medium. Concentrations in the microdialyis samples were determined initially (in $\mathrm{pg} / \mathrm{ml}$ ) of the neurotransmitter from a regression line fitted to ACh concentrations of the calibration samples versus the corresponding area mass to charge ratios $(\mathrm{m} / \mathrm{z})$ of the selected-reaction monitoring-chromatographic peaks for $\mathrm{ACh}(\mathrm{m} / z 146 \rightarrow 87)$ and $\mathrm{ACh}-\mathrm{d}_{4}(\mathrm{~m} / z 150 \rightarrow 91)$. For statistical analyses and charting the results, we expressed ACh levels as percentage of its mean baseline concentration.

Immunohistochemistry. To verify that the optical stimulation activated cholinergic neurons and to identify the probe location, we stimulated the $\mathrm{BF}$ with a $10 \mathrm{~s}$ train of $10 \mathrm{~Hz}$ light stimulation every $60 \mathrm{~s}$ for $2 \mathrm{~h}$ from ZT 2 to ZT 4 and then performed immunohistochemistry for ChAT and for c-Fos protein, a marker of neuronal activation. Immediately after completion of optical stimulation, the mice were perfused transcardially with saline and a $10 \%$ formalin solution. Perfused brains were removed, postfixed overnight in $10 \%$ formalin, and then immersed in a $30 \%$ sucrose solution at $4^{\circ} \mathrm{C}$ for cryoprotection. $40 \mu \mathrm{m}$-thick coronal slices were collected in 1 of 4 wells (series), and stored in PBS at $4^{\circ} \mathrm{C}$. For all antibody stains, control stains were performed in slices where primary antibodies were omitted. For ChAT/cFos protein colocalization, sections from one well were washed in PBS and treated with $0.2 \%$ Triton-X for $2 \mathrm{~h}$, and blocked using 3\% normal donkey serum for $1 \mathrm{~h}$. After blocking, rabbit polyclonal primary antibody, anti-c-Fos (1:2000 dilution, Santa Cruz Biotechnology, SC-253) was added and incubated overnight on a platform shaker. On the next day, slices were washed and treated with the secondary antibody, donkey anti-rabbit conjugated to AlexaFluor-568, red (1:2000; Invitrogen, A10042) for $2 \mathrm{~h}$. Next, the sections were washed in PBS and incubated overnight with the second primary antibody, a goat polyclonal anti-ChAT antibody (1:500; Millipore Bioscience Research Reagents, AB144). The following day, the sections were washed and incubated with donkey anti-goat IgG conjugated to AlexaFluor-488, green (1:500; Invitrogen, A21467) for $2 \mathrm{~h}$ at room temperature. Imaging of cells at higher magnifications $(40 \times)$ were performed using a Zeiss LSM confocal microscope. To determine the number of cholinergic neurons that are activated by optical stimulation, we counted the number of ChATcFos-positive neurons in the BF on both sides of the brain (ipsilateral to opto-dialysis probe and contralateral side as control). In three mice, three sections from each mouse between bregma $0.2 \mu \mathrm{m}$ and $-0.2 \mu \mathrm{m}$ ( 1 in 4 series) were used for counting.

Statistical analyses. All values are shown as mean \pm SEM. Statistical analyses were performed using SigmaPlot (version 11.0, Systat Software). For comparisons between two time points within the same group of animals, we used paired $t$ tests. In situations when the normality assumption failed, a Wilcoxon signed rank test was used. For analyses involving several time-points and or multiple factors, we used one-way or two-way repeated-measures ANOVA. All repeated-measures ANOVAs were followed by Holm-Sidak post hoc tests.

\section{Experimental protocols}

Experiment 1: testing the opto-dialysis probe. In our first set of experiments, we confirmed that the presence of the microdialysis probe did not prevent optical stimulation and then determined the effect of optical stimulation on BF cholinergic activity and behavioral state. We initially used a stimulation frequency of $10 \mathrm{~Hz}$ modeled after the firing pattern of identified cholinergic neurons during wakefulness (Lee et al., 2005) and previously tested in our in vitro study (Yang et al., 2014) in the same strain of mice constitutively expressing ChR2 in cholinergic neurons (Zhao et al., 2011). Experiments were done at ZT 2-4 (i.e., during the natural sleeping period when ACh levels are normally low) (Vazquez and Baghdoyan, 2001). To investigate the effect of optogenetic stimulation of cholinergic neurons on local ACh release surrounding the target of optical stimulation in the BF, microdialysis samples for subsequent measurement of ACh were collected on a No-Stimulation day and on a Stimulation day. EEG was monitored throughout the experiment. After a $1 \mathrm{~h}$ same-day baseline period, optical stimulation was performed from ZT 2 to ZT 4 on the Stimulation day, consisting of $10 \mathrm{~s}$ trains of $10 \mathrm{~Hz}$ pulses with a pulse width of $10 \mathrm{~ms}$ delivered every minute for $2 \mathrm{~h}$. On the No-Stimulation day, the experiment was conducted in the same way, except that the laser was not turned on. The time spent in wakefulness, NREM sleep, and REM sleep was determined during $2 \mathrm{~h}$ of optical stimulation and compared with that of the time matched no-stimulation day. To compare the changes in extracellular ACh levels during optical stimulation with the changes in ACh occurring during prolonged wakefulness, the mice were sleep-deprived (SD) for $3 \mathrm{~h}$ (ZT 2-5) on a different day, by introducing novel objects into the cage and gently tapping the cage when necessary to prevent sleep. Samples from the SD day were collected as a positive control because cholinergic neurons discharge at a higher rate during wakefulness (Lee et al., 2005); consequently, the level of ACh in the BF is higher compared with NREM sleep (Vazquez and Baghdoyan, 2001).

Experiment 2: testing multiple stimulation frequencies. The discharge frequency of cholinergic neurons varies across sleep/wake states with the highest mean discharge rate during REM sleep $(16.3 \mathrm{~Hz})$, a lower rate of $7.6 \mathrm{~Hz}$ during wakefulness, and a greatly reduced rate in NREM sleep $(0.84 \mathrm{~Hz})$ (Lee et al., 2005). While our experiments described above showed that $10 \mathrm{~Hz}$ stimulation was effective in increasing wakefulness, we wanted to systematically evaluate which stimulation frequency resulted in the highest increase in wakefulness. Thus, next we tested other stimulation frequencies and train durations $(2,5,8$, and $10 \mathrm{~Hz}$ for $5 \mathrm{~s}$ and 
8 and $10 \mathrm{~Hz}$ for $10 \mathrm{~s}$ ) within the range of discharge rates of cholinergic neurons observed during wakefulness and NREM sleep. For each frequency, a train of $5 \mathrm{~s}$ was repeated every minute for $2 \mathrm{~h}$ starting at ZT 2. For 8 and $10 \mathrm{~Hz}$, in addition to $5 \mathrm{~s}$ trains, a $10 \mathrm{~s}$ train of the selected frequency was repeated every minute for $2 \mathrm{~h}$. The amount of wakefulness on each Stimulation day was compared with a time-matched baseline day without optical stimulation (No-Stimulation) day. These experiments were performed in the same animals and thereby also ensured that the effect of optical stimulation of BF cholinergic neurons on wakefulness was repeatable and not altered by multiple rounds of stimulation.

Experiment 3: $8 \mathrm{~Hz}$ BF cholinergic stimulation and reverse microdialysis perfusion of cholinergic receptor antagonists. To explore the possible role of local ACh release in the BF on the wake-promoting effect of cholinergic BF stimulation, we used the opto-dialysis system to locally perfuse the muscarinic receptor antagonist, atropine (50 $\mu \mathrm{M}$ diluted in aCSF; WestWard Pharmaceuticals), or a combination of atropine and mecamylamine (nicotinic receptor antagonist; $1 \mathrm{~mm}$ diluted in aCSF; Sigma) during the $2 \mathrm{~h}$ optical stimulation period on the No-Stimulation and Stimulation day. The concentration of the antagonists was determined based on the doses shown to be effective in blocking cholinergic effects on GABAergic neurons in our previous in vitro study (Yang et al., 2014) and an assumption that the $1 \mathrm{~mm}$ microdialysis probe allows $\sim 10 \%$ of the drug to reach the target site (Porkka-Heiskanen et al., 2000). After a $1 \mathrm{~h}$ same-day baseline period, optical stimulation was performed from ZT 2 to ZT 4 on the Stimulation day and consisted of $10 \mathrm{~s}$ trains of $8 \mathrm{~Hz}$ pulses with a pulse width of $10 \mathrm{~ms}$ delivered every minute for $2 \mathrm{~h}$.

\section{Results}

Optical stimulation of BF cholinergic neurons increased c-Fos expression, wakefulness, and $\mathrm{BF}$ acetylcholine release

Optical stimulation of cholinergic neurons $(10 \mathrm{~Hz}$ for $10 \mathrm{~s} / \mathrm{min}$ for $2 \mathrm{~h}$ ) followed by post hoc immunohistochemistry revealed that the protein product of the immediate-early gene, $c$-Fos, a widely used marker of neuronal activation was expressed in the nuclei of $58.6 \pm 3.6 \%$ of $\mathrm{ChAT}^{+}$neurons on the side ipsilateral to the probe (Fig. 2a). This was significantly higher $(p<0.001, n=3)$ than $5.3 \pm 1.1 \%$ of the $\mathrm{ChAT}^{+}$neurons with nuclear $c$-Fos observed on the contralateral side, thus confirming that, as expected, optical stimulation activated cholinergic neurons. Analysis of behavioral state in these experiments revealed that during the $2 \mathrm{~h}$ stimulation period, the time spent in wakefulness was significantly increased by $51 \pm 24 \%$ (paired $t$ test, $t_{(4)}=-2.935, p=0.043$ ), whereas NREM sleep was significantly decreased by $17 \pm 8 \%$ (paired $t$ test, $t_{(4)}=2.968, p=0.041$ ) compared with the time-matched period on the No-Stimulation day (Fig. 2b). There was no significant effect on REM sleep (REM sleep decreased by $13 \pm 23 \%$, paired $t$ test, $t_{(4)}=0.672, p=$ $0.538)$. Concurrent with the increase in wakefulness produced by optogenetic stimulation of BF cholinergic neurons, we observed an increase in BF ACh levels, measured using the microdialysis component of the opto-dialysis probe in combination with LCMS/MS (Fig. 2c). There were no differences in the absolute levels of ACh during the baseline periods on the No-Stimulation day $(74.95 \pm 19.2 \mathrm{pg} / \mathrm{ml})$ and Stimulation day $(76.44 \pm 33.85 \mathrm{pg} / \mathrm{ml})$ in the same animals $(N=5)$. Extracellular BF ACh levels were significantly increased during the first $(46.11 \pm 11.8 \%)$ and the second hour $(66.09 \pm 12.8 \%)$ of optical stimulation of BF cholinergic neurons, compared with the prestimulation baseline (set to $100 \%$, indicated by Fig. $2 d$, horizontal line). Time of the day comparison with No-Stimulation day showed that optical stimulation significantly increased ACh levels by $81 \pm 14 \%$ (paired $t$ test, $t_{(4)}=-3.962, p=0.017$ ) and by $86 \pm 14 \%$ (paired $t$ test, $\left.t_{(4)}=-3.794, p=0.019\right)$ during the first (ZT 2-3) and second (ZT 3-4) hour, respectively.
We also compared the optogenetically evoked increase in ACh to the physiological increase induced by sleep deprivation (SD, Fig. 2e). The average increase in ACh levels during the $2 \mathrm{~h}$ of optical stimulation $\left(83.5 \pm 14 \%\right.$, paired $t$ test, $t_{(4)}=-5.080, p=$ 0.007 ) was comparable (no significant difference; $n=5$, one-way repeated-measures ANOVA) to the increase observed during $3 \mathrm{~h}$ of SD $(69 \pm 13 \%$, Wilcoxon signed rank test, $p=0.031)$. Thus, optical stimulation increases ACh levels in the BF close to the maximal physiological levels. The levels returned to baseline values at the cessation of stimulation, similar to the decreases observed during recovery sleep following SD (Fig. $2 d, e$ ).

Together, these experiments were demonstrative of the success of our combined opto-dialysis probe design and its use in conjunction with EEG/EMG assessments of behavioral state.

\section{Behavioral response to multiple stimulation frequencies}

To determine the optimal stimulation frequency to elicit the maximal effect on wakefulness, we used a counterbalanced design, whereby on different days, stimulation frequencies of 2, 5, 8, or $10 \mathrm{~Hz}$ were tested. We observed that stimulation at all frequencies, other than $2 \mathrm{~Hz}$, led to a significant increase in wakefulness during the $2 \mathrm{~h}$ stimulation period. However, in a repeatedmeasures ANOVA versus control group (post hoc Holm-Sidak), the $8 \mathrm{~Hz} 10 \mathrm{~s}$ and $10 \mathrm{~Hz} 10 \mathrm{~s}$ stimulation protocols were the only protocols to reach a significant difference from the NoStimulation day. Although not significantly different from the effects of $10 \mathrm{~Hz}$ stimulation the absolute largest increase in wakefulness was observed during optical stimulation at $8 \mathrm{~Hz}, 10 \mathrm{~s} / \mathrm{min}$ for $2 \mathrm{~h}$ (paired $t$ test, $t_{(4)}=-3.856, p<0.05$; Fig. $3 a$ ). Thus, we used this paradigm of $8 \mathrm{~Hz}$ stimulation for $10 \mathrm{~s} / \mathrm{min}$ for all subsequent experiments and analyses. Using this paradigm, power spectral analysis of wakefulness periods (averaged over the $2 \mathrm{~h}$ stimulation period) on the Stimulation day showed no significant difference compared with periods of spontaneous wakefulness on the No-Stimulation day $(n=6 ; 0.25 \mathrm{~Hz}$ bins; two-way repeated-measures ANOVA, $p>0.05$; Fig. $3 b$ ). This finding suggested that optical stimulation of BF cholinergic neurons caused an increase in physiological wakefulness. There was a trend toward increased $\theta(4-8 \mathrm{~Hz})$ activity, consistent with the results of a previous pharmacological study, which selectively stimulated BF cholinergic neurons (Cape et al., 2000). However, this trend did not reach statistical significance. Very few stimulations (2.8 \pm $0.9)$ resulted in NREM to REM transitions during $2 \mathrm{~h}$ stimulation period, which was similar to the number of NREM to REM transitions observed on time-matched No-Stimulation day (1.6 \pm $0.5 ; p=0.28, n=5$; paired $t$ test, $\left.t_{(4)}=-1.238, p=0.284\right)$.

\section{Cholinergic stimulation increased the probability and decreased the latency for transitions from NREM to wakefulness}

Optical stimulation of BF cholinergic neurons at $8 \mathrm{~Hz}$ resulted in a $84 \pm 9 \%$ increase in wakefulness compared with the same time on the No-Stimulation day (paired $t$ test, $t_{(5)}=-3.817, p=$ 0.012 ; Fig. $4 a$ ). The probability of NREM to wake transitions increased, especially in the first $10 \mathrm{~s}$ after the start of stimulation (298 $\pm 74 \%$ increase in transitions from NREM sleep to wake, paired $t$ test, $t_{(5)}=-3.579, p=0.016$; Fig. $4 b-d$ ). The mean latency for NREM sleep to wake of $25.6 \mathrm{~s}$ on the baseline NoStimulation day decreased to $13.5 \mathrm{~s}$ on the stimulation day (Wilcoxon signed rank test, $p<0.001$; Fig. $4 c$ ). 
a

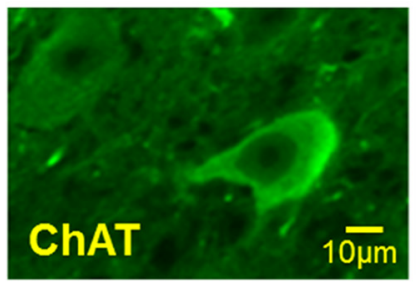

b
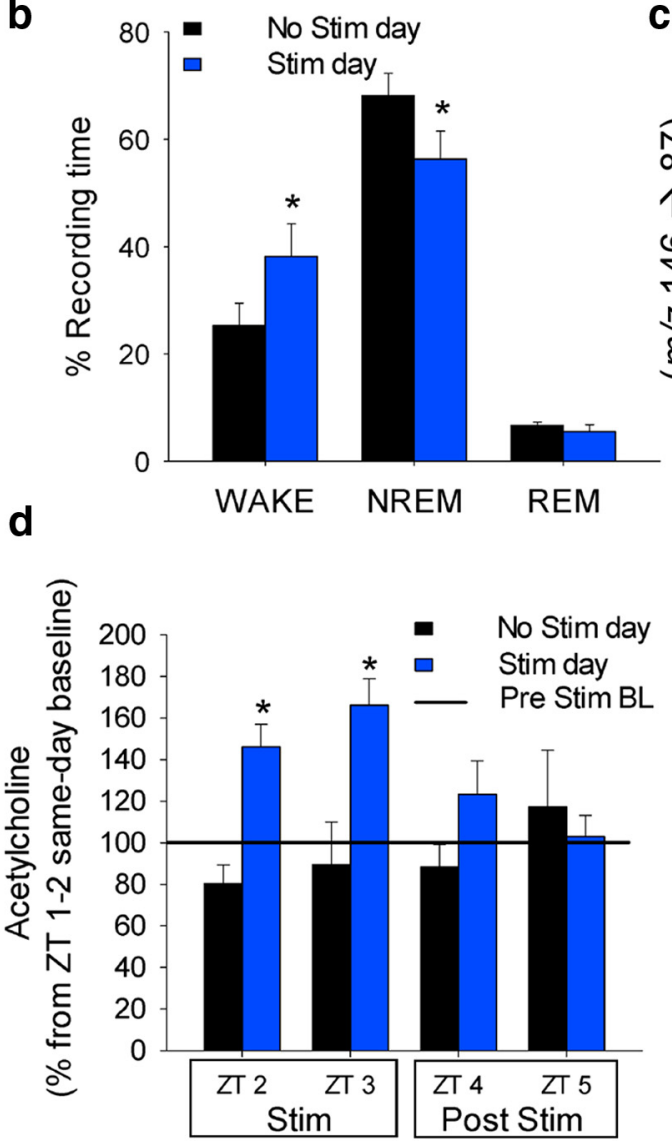

C
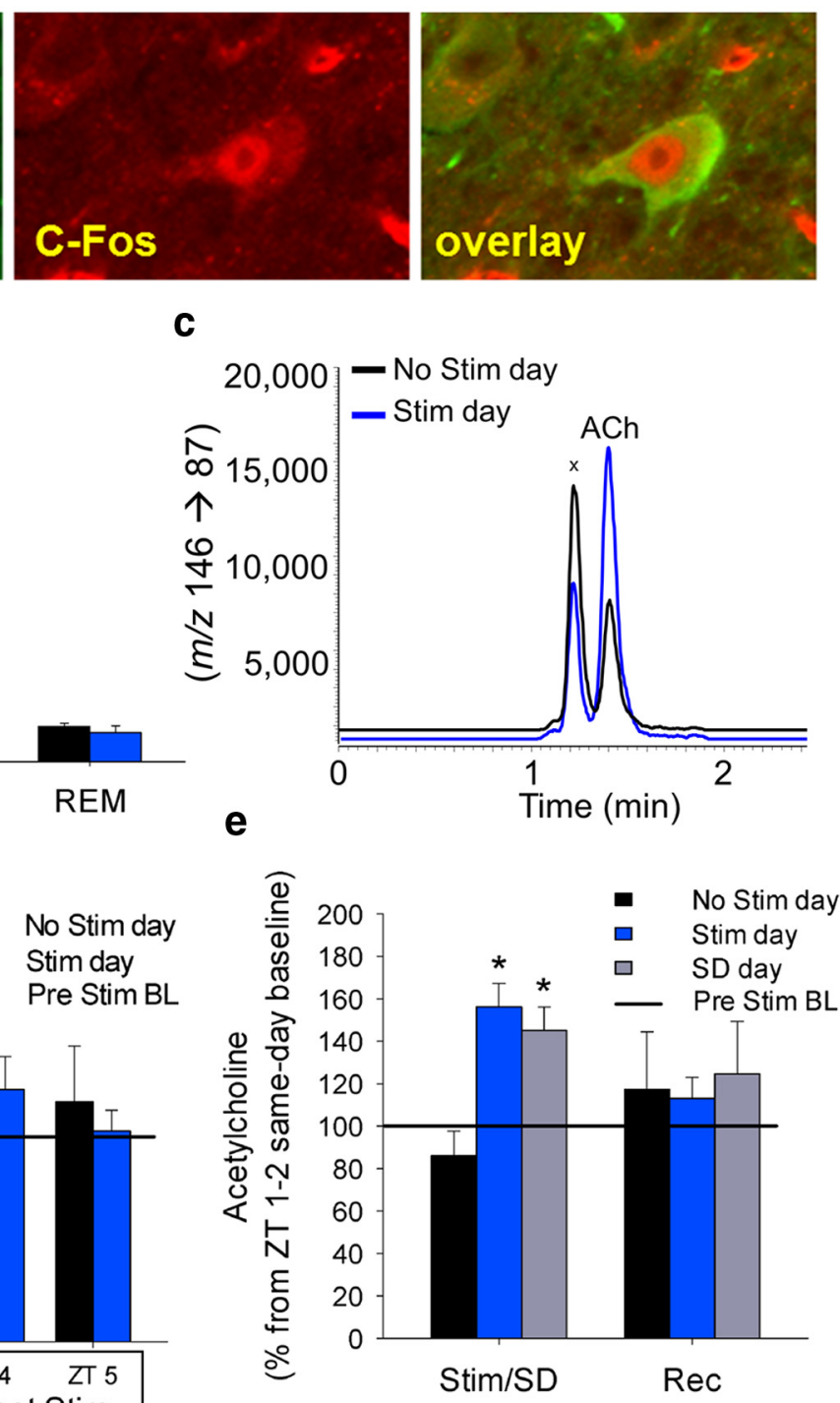

Figure 2. Optogenetic stimulation of BF cholinergic neurons activates cholinergic neurons, increases wakefulness, and increases local ACh levels surrounding the opto-dialysis probe. $\boldsymbol{a}$, Optogenetic stimulation activates BF cholinergic neurons (green, stained with ChAT). Staining of the immediate early gene product, c-Fos (red) illustrates neuronal activation in the nuclei of BF cholinergic neurons (overlay). $\boldsymbol{b}$, The percentage of wakefulness significantly increased and NREM sleep decreased during the $2 \mathrm{~h}$ optogenetic stimulation period, compared with the same time period on the baseline (No Stim) day. c, Representative traces of LC-MS/MS measurements showing the increase in local BF ACh levels (mass to charge ratio, m/z) produced by optogenetic stimulation. ${ }^{\mathbf{x}}$ unknown constituent of the microdialysates that generates an isobaric ion with ACh but separated from ACh chromatographically. $\boldsymbol{d}$, Extracellular BF ACh levels were significantly increased during the first and the second hour of optical stimulation of BF cholinergic neurons, compared with the baseline (No Stim) day and compared with the prestimulation baseline (BL, set to $100 \%$, indicated by the horizontal line). ACh levels returned to BL levels immediately after stimulation ended. $\boldsymbol{e}$, The increased ACh levels caused by optogenetic stimulation are comparable with levels seen during a $3 \mathrm{~h}$ sleep deprivation (SD) period. Optogenetic stimulation paradigm: $10 \mathrm{~Hz}, 10$ s repeated every minute for $2 \mathrm{~h} ; n=6$ (BL), 5 (Stim/SD). ${ }^{*} p<0.05$ (paired $t$ test/one-way repeated-measures ANOVA post hoc Holm-Sidak). Data are mean \pm SEM). Stim, Stimulation.

Reverse microdialysis of ACh receptor antagonists blocked the increase in wakefulness produced by optical stimulation of cholinergic neurons

Although BF cholinergic stimulation was effective in promoting transitions to wakefulness, it was notable that the latency to wakefulness was prolonged (mean latency 13.5) compared with the effect of optogenetic stimulation of other neurotransmitter systems (e.g., noradrenergic locus ceruleus neurons, $<5 \mathrm{~s}$ ) (Carter et al., 2010), suggesting that an intermediate step may be required. Our microdialysis experiments showed that local ACh levels were increased by optical stimulation and, as discussed above, cholinergic neurons activate neighboring non-cholinergic neurons (Yang et al., 2014; Xu et al., 2015), which may also play a role in cortical activation (Duque et al., 2000; Manns et al., 2003; Lin et al., 2006; Anaclet et al., 2015; Xu et al., 2015). Thus, in our final series of experiments, we investigated the functional effects of increased BF ACh levels on sleep and wakefulness.

Using our newly designed opto-dialysis system, we locally applied the muscarinic receptor antagonist, atropine, during optical stimulation, by reverse microdialysis. Strikingly, application of atropine $(50 \mu \mathrm{M})$ blocked the increase in wakefulness and decrease in NREM sleep produced by stimulation of cholinergic neurons (Fig. 5). Application of atropine to the BF during a baseline No-Stimulation day had no effect on sleep-wake states (Stimulation day significantly different from: No-Stimulation baseline day, No-Stimulation day + atropine, and Stimulation day + atropine, $n=6$, one-way repeated-measures ANOVA, post hoc Holm-Sidak, Wake: $F_{(5,14)}=9.393$, NREM: $F_{(5,14)}=10.038$, 


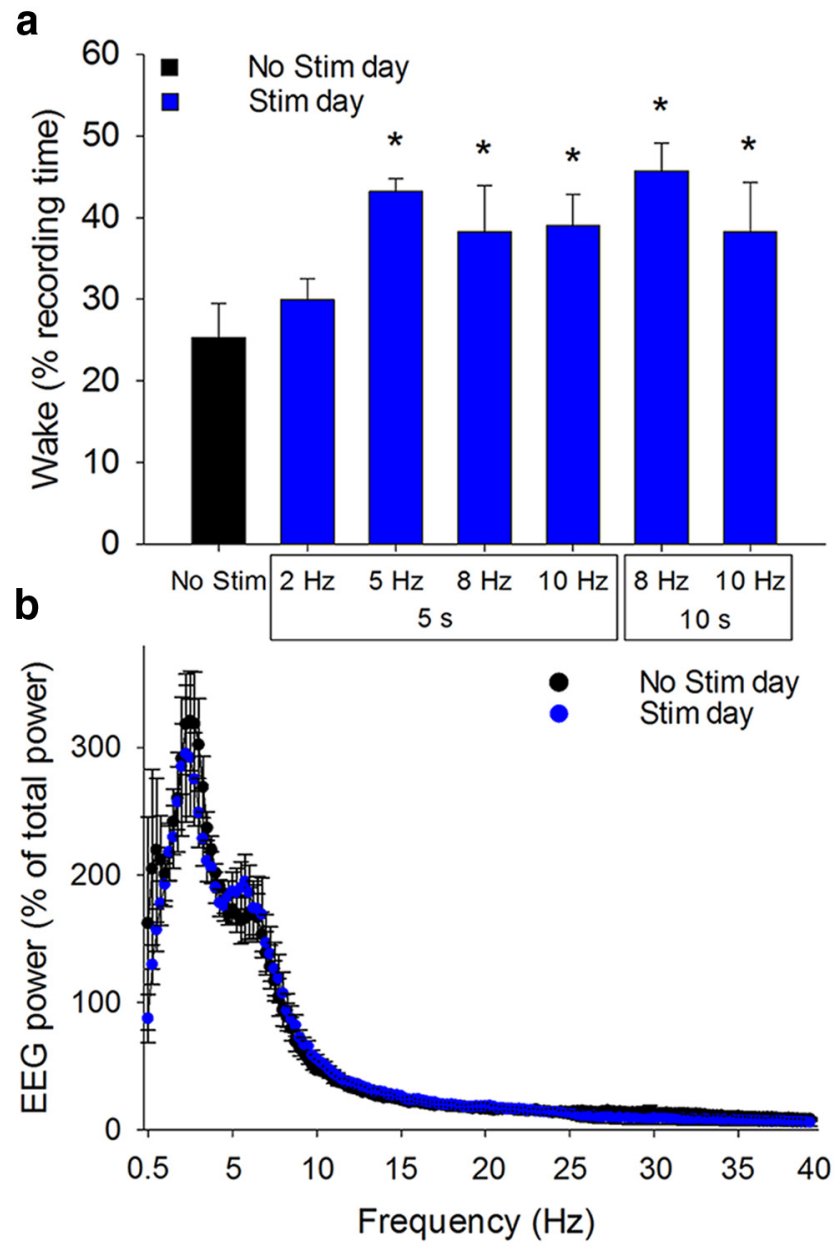

Figure 3. Increased wakefulness induced by optogenetic stimulation of BF cholinergic neurons has a similar spectral profile to spontaneous wakefulness. $\boldsymbol{a}$, The total amount of wakefulness increased during the stimulation period when $\mathrm{BF}$ cholinergic neurons were stimulated at 5 , 8 , or $10 \mathrm{~Hz}$; but not at $2 \mathrm{~Hz}$. The largest effect on wake was seen at $8 \mathrm{~Hz} 10 \mathrm{~s}$. This frequency and duration were used for all further experiments $(n=5) .{ }^{*} p<0.05$ (paired $t$ test). Data are mean \pm SEM. $\boldsymbol{b}$, During the $2 \mathrm{~h}$ stimulation period on the baseline (No Stim) day and Stim day, power spectra of wakefulness periods were not significantly different, indicating that induced wakefulness was not different from spontaneous wakefulness ( $n=6 ; 0.25 \mathrm{~Hz}$ bins). EEG power expressed as percent of total power $(0.5-20 \mathrm{~Hz})$ during the same experiment day for each animal. $p>0.05$ (two-way repeated-measures ANOVA).

$p<0.001$; Fig. 5a). The probability of NREM sleep to wake transitions decreased when optical stimulation was combined with simultaneous atropine perfusion, compared with optical stimulation alone, and the mean latency to wakefulness increased (Optical stimulation, $13.5 \mathrm{~s}$; Stimulation + atropine, $20.9 \mathrm{~s}$ ). Although both measures were significantly different compared with optical stimulation alone $(n=6$, one-way repeated-measures ANOVA, post hoc Holm-Sidak, transition to wake within $10 \mathrm{~s}$ : $F_{(5,13)}=9.863, p=0.001$, mean latency: $F_{(5,13)}=9.189, p=$ 0.002 ) and did not significantly differ from the No-Stimulation day, a small residual effect of stimulation remained (Fig. $5 b-d$ ). Reverse microdialysis of a mixture of muscarinic and nicotinic ACh receptor antagonists, atropine and mecamylamine $(50 \mu \mathrm{M}$ and $1 \mathrm{~mm}$, respectively) further decreased the probability and increased the latency of NREM sleep to wake transitions (23.95 s) to values comparable (not statistically different, $n=4$, one-way repeated-measures ANOVA, mean latency: $p=0.81$ ) with those on the drug-free baseline No-Stimulation day (Fig. $5 b-d$ ).

\section{Discussion}

In this report using our novel opto-dialysis probe, we performed combined optical manipulation and local in vivo microdialysis, simultaneously in freely behaving mice. We observed that the optical stimulation of BF cholinergic neurons increased local ACh levels and increased wakefulness. Blocking the effects of ACh by reverse microdialysis of cholinergic antagonists within the BF prevented the wake-promoting effect of cholinergic stimulation. Thus, our results present evidence for a paradigm shift in our understanding of BF control of cortical activity, challenging the prevailing dictum that BF cholinergic activity directly promotes wakefulness via projections to the cortex.

\section{Design of a novel combined opto-dialysis probe}

To be useful, a combined opto-dialysis probe must fulfill certain requirements. First, the probe must be small enough and stable enough to be implantable in the mouse brain without significant damage to the brain and must allow normal behavior. Indeed, our opto-dialysis probe is sized to fit the mouse brain, allows implantation of EEG electrodes and free movement of the mouse. Baseline recordings confirmed that the distribution of sleepwake states was similar to those in wild-type animals. Second, the microdialysis probe must not interfere with the function of the fiber optic cannula and vice versa. Our experiments here show that optical stimulation of cholinergic neurons increased wakefulness and increased the ACh concentration to levels similar to those measured during sleep deprivation, and thus are consistent with this second requirement.

Using this device, our data represent the first in vivo demonstration of the local release of ACh in response to the optical stimulation of BF cholinergic neurons. Previous work performed in rats describes the use of reverse microdialysis of pharmacological agents during optical stimulation (Taylor et al., 2014). Although technically impressive, this study necessitated the use of a large implant assembly consisting of a separate optical probe and microdialysis cannula. Additionally, technically challenging angular implantation techniques were required to ensure proper placement of the optical fiber tip adjacent to the microdialysis probe. Thus, the opto-dialysis probe described here represents a significant technological advance in its combination of the optic fiber cannula and microdialysis into a single unit suitably sized for work in mice.

A potential drawback of optogenetic stimulation is that synchronized activation of the targeted neuronal population might raise neurotransmitter concentrations to unphysiological levels. However, the observed 83\% increase in ACh levels in this study was not significantly different from the physiological 69\% increase we observed during sleep deprivation. Additionally, cessation of optical stimulation decreased ACh values to basal levels, as was also observed during recovery sleep after prolonged wakefulness (Fig. 2e). We conclude that our optical stimulation paradigm induced variations in ACh concentration and kinetics comparable with state-dependent changes reported previously in BF (Vazquez and Baghdoyan, 2001).

\section{Optical stimulation of BF cholinergic neurons promotes NREM-wake transitions}

In accord with three reports on the arousal effects of optical stimulation of BF cholinergic neurons using conventional optogenetics (Han et al., 2014; Irmak and de Lecea, 2014; Xu et al., 2015), $8-10 \mathrm{~Hz}$ stimulation increased time spent in wakefulness during $2 \mathrm{~h}$ of optical stimulation compared with the time-matched pe- 
a
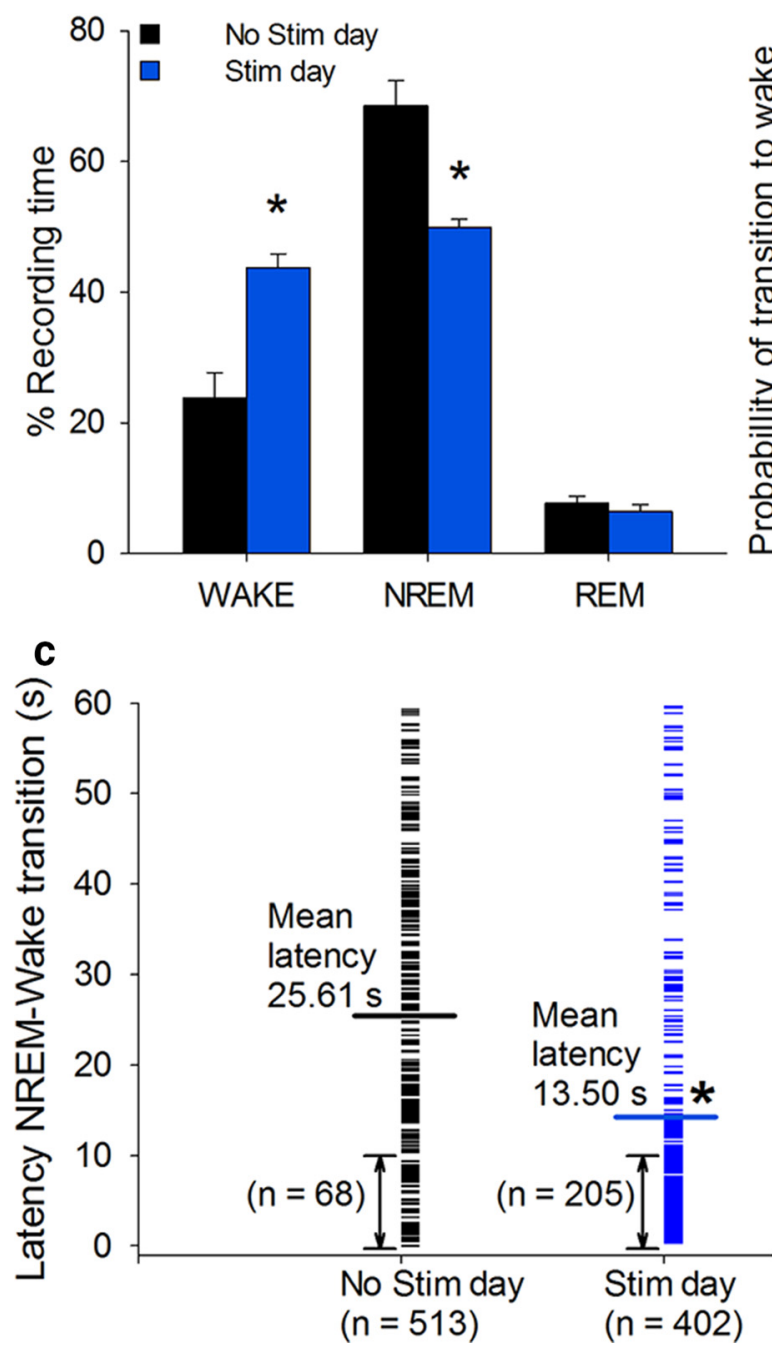

b

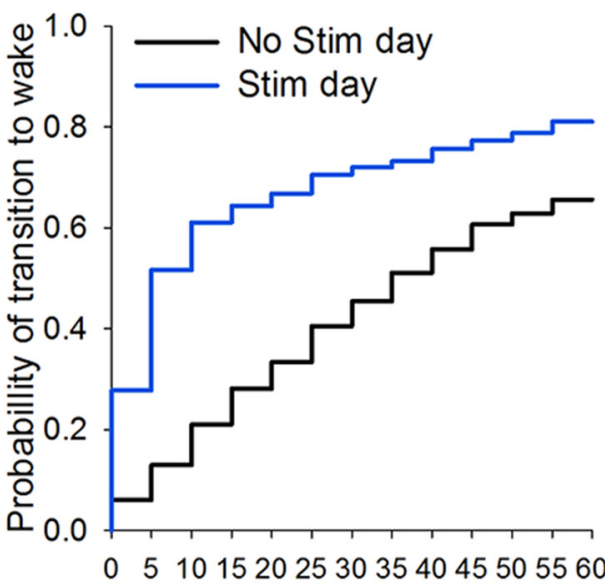

d $\quad$ Time $(\mathrm{s})$

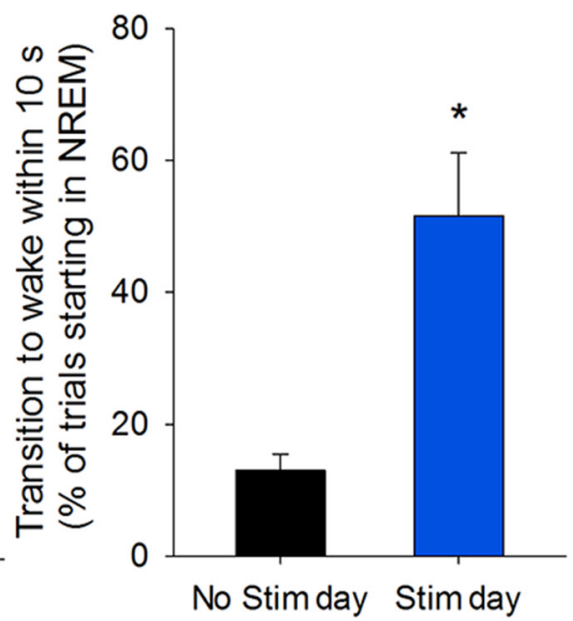

Figure 4. Optogenetic stimulation of BF cholinergic neurons increases wakefulness, increases the probability of NREM to wake transitions, and reduces the latency to wakefulness. $\boldsymbol{a}, 0$ ptogenetic stimulation $(8 \mathrm{~Hz}, 10 \mathrm{~s} / \mathrm{min}, 2 \mathrm{~h}$ ) increased wakefulness and decreased NREM sleep compared with the baseline (No stim) day. $\boldsymbol{b}$, The cumulative probability distribution of NREM sleep to wakefulness transition latencies shows an increased probability to wake on the Stim day compared with the baseline day (513 and 402 trials starting in NREM for No Stim and Stim conditions, respectively). $\boldsymbol{c}$, NREM sleep to wake latency decreased during the stimulation period compared with the same period on the baseline day. $\boldsymbol{d}$, Transitions from NREM sleep to wakefulness within $10 \mathrm{~s}$ of the start of stimulation increases compared with the baseline day $(n=6) .{ }^{*} p<0.05$ (paired $t$ test/Wilcoxon signed rank test). Data are mean \pm SEM.

riod on the No-Stimulation day. Our parametric experiments showed that $8 \mathrm{~Hz}$ stimulation was most effective in inducing wakefulness, similar to the results of Irmak and de Lecea (2014). Spectral analysis confirmed that the wake-EEG parameters during the $2 \mathrm{~h}$ of optical stimulation period remained unchanged, compared with natural wakefulness, suggesting that optical stimulation induced transitions to a physiological state of wakefulness.

Cholinergic stimulation facilitated the probability of NREM to wakefulness transitions and decreased their latency as reported previously (Han et al., 2014; Irmak and de Lecea, 2014; Xu et al., 2015). In our study, optical stimulation of BF cholinergic neurons reduced the mean latency to wakefulness by $\sim 50 \%$ (to $13.5 \mathrm{~s})$ compared with the average latency observed on the NoStimulation day (TTL pulse without laser, $25.6 \mathrm{~s}$ ). This value is lower than the latencies reported $(\sim 25-45 \mathrm{~s})$ by Irmak and de Lecea (2014). This difference in the latency may be attributable to the percentage of cholinergic neurons expressing ChR2 and to the basal cholinergic tone in the mouse models used in the two studies. Irmak and de Lecea (2014) used ChAT-Cre mice in com- bination with AAV-ChR2-EYFP viral injections to transduce $\sim 64 \%$ of the BF cholinergic neurons. Han et al. (2014) used a BAC transgenic strain of ChAT-ChR2-EYFP mice with constitutive $\mathrm{ChR} 2$ expression in $>90 \%$ of $\mathrm{BF}$ cholinergic neurons (Yang et al., 2014). This is the same strain of mice used in our work, and its increased cholinergic tone (Kolisnyk et al., 2013) may account for the shorter observed state transition latencies $(<15 \mathrm{~s})$ that we and Han et al. (2014) found, in contrast to the findings of Irmak and de Lecea (2014). It is unlikely that the difference in latency is due to stimulation of brainstem cholinergic neurons since in the ChAT-ChR2 mice line used in this study the expression of ChR2EYFP was minimal in the brainstem cholinergic neurons (Yang et al., 2014).

The wake-promoting effect of BF cholinergic stimulation requires activation of neighboring non-cholinergic neurons The most important biological result of this study is that simultaneous reverse microdialysis of ACh receptor antagonists into BF prevented the increased NREM to wakefulness state transitions in response to light stimulation, suggesting that 

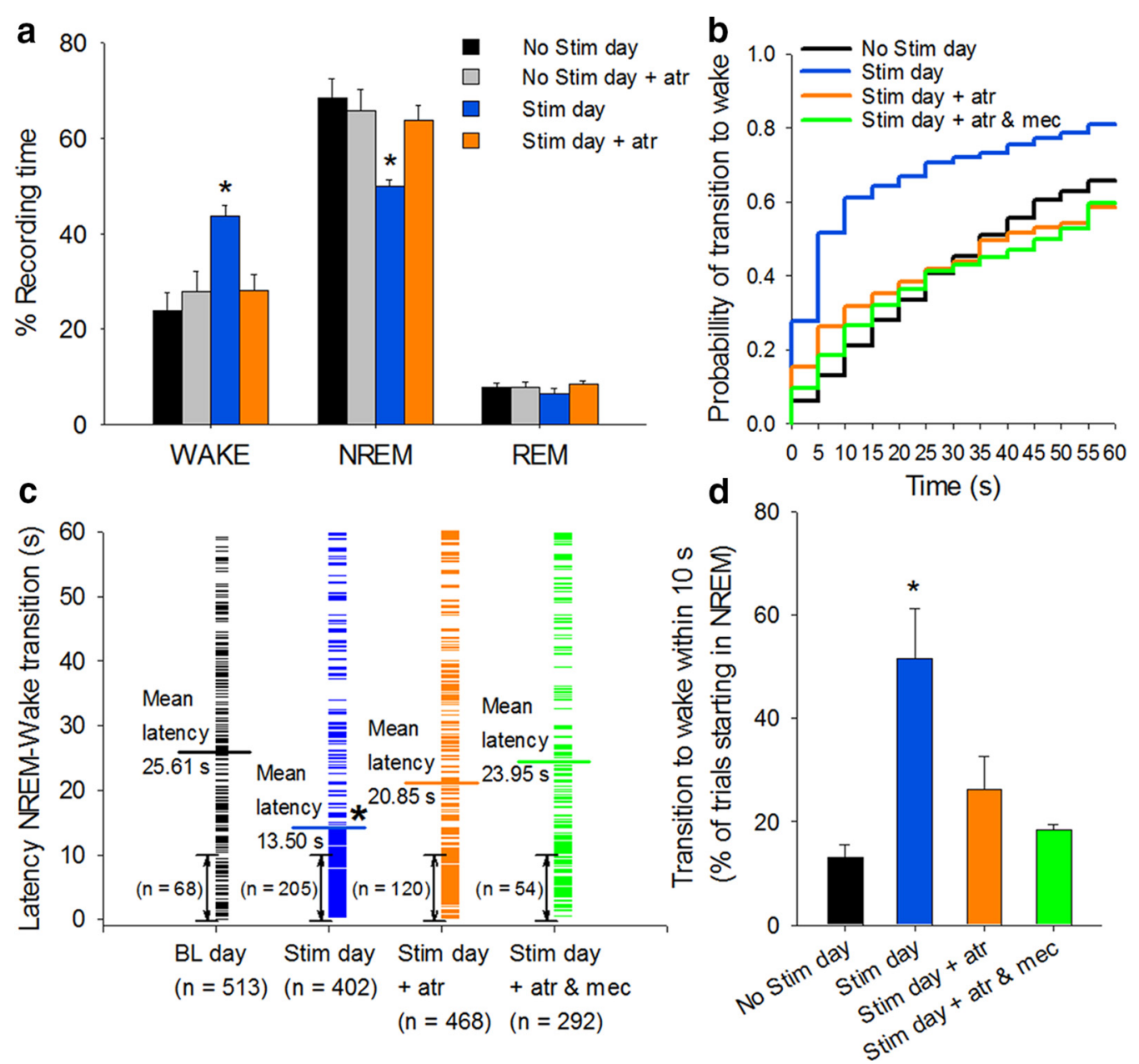

Figure 5. The wake-promoting effect of cholinergic stimulation is strongly attenuated by simultaneous reverse microdialysis of cholinergic receptor antagonists into the BF. Reverse microdialysis of atropine (atr, $50 \mu \mathrm{M})$, or a mixture of atropine $(50 \mu \mathrm{m})$ and mecamylamine (mec, $1 \mathrm{~mm}$ ) into the BF during optogenetic stimulation of cholinergic neurons abolishes the effect on the following: $\boldsymbol{a}$, wakefulness; $\boldsymbol{b}$, probability of NREM sleep to wakefulness transitions; $\boldsymbol{c}$, NREM sleep to wakefulness latency; and $\boldsymbol{d}$, transitions from NREM sleep to wakefulness within $10 \mathrm{~s}$ of the start of stimulation (Stim + atr, $n=6$; Stim day + atr and mec, $n=4$ ). ${ }^{*} p<0.05$ (one-way repeated-measures ANOVA post hoc Holm-Sidak). Data are mean \pm SEM.

these state transitions require local ACh activation of neighboring non-cholinergic, wakefulness-promoting neurons. Lesion experiments suggest that both cholinergic and noncholinergic neurons in $\mathrm{BF}$ are required for cortical arousal (Semba, 2000; Kaur et al., 2008; Fuller et al., 2011). Among the non-cholinergic neurons, neuroanatomical (Freund and Meskenaite, 1992) and electrophysiological recording (Duque et al., 2000; Henny and Jones, 2008; Hassani et al., 2009; McKenna et al., 2013) experiments suggested that BF GABAergic neurons may be particularly important in controlling cortical activation and wakefulness. Recent experiments using sloweracting, pharmacogenetic techniques suggested that activation of BF GABAergic neurons strongly promotes wakefulness, whereas inhibition promoted sleep (Anaclet et al., 2015). In contrast, the effect of pharmacogenetic activation of BF glutamatergic or cholinergic neurons on wakefulness was less pronounced. Furthermore, optogenetic studies using both gainand loss-of-function experiments provided compelling evidence that BF GABAergic/PV neurons control cortical fast activity typical of wakefulness (Kim et al., 2015). Cholinergic terminals from local collaterals and brainstem cholinergic neurons are located in close proximity of BF non-cholinergic neurons (Martinez-Murillo et al., 1990; Zaborszky and Duque, 2000; Duque et al., 2007). Specifically, cholinergic fi- ber varicosities appose GABAergic/PV neurons in BF (Yang et al., 2014). In vitro studies from our group demonstrated an excitatory effect of the cholinergic agonist carbachol on identified BF GABAergic and PV neurons (Yang et al., 2014). Furthermore, in vitro optical stimulation of cholinergic neurons in the same line of ChAT-ChR2-EYFP mice used here reliably elicited an inward current in non-cholinergic neurons with the same intrinsic membrane properties as cortically projecting GABAergic/PV neurons (Yang et al., 2014). Xu et al. (2015) confirmed these excitatory effects of cholinergic neurons on BF PV neurons using optogenetic techniques. Thus, these experiments suggest that cholinergic stimulationinduced state transitions from NREM to wakefulness are mediated via local ACh effects on the activity of cortically projecting BF GABAergic neurons, including the subset that contains PV. Although optogenetic stimulation of BF glutamatergic (vesicular glutamate transporter type 2, vGluT2 ${ }^{+}$) neurons promoted wakefulness (Xu et al., 2015), pharmacological or optogenetic stimulation of the BF cholinergic system in vitro strongly inhibited vGluT2 ${ }^{+}$neurons in vitro (Xu et al., 2015; Yang et al., 2015), suggesting that local activation of BF glutamatergic neurons is an unlikely mediator of the wakepromoting effects of cholinergic stimulation. The optodialysis technique described here will be useful in the future 
for disentangling local versus distal effects of stimulation of BF GABAergic and vGluT2 ${ }^{+}$neurons.

In conclusion, this report demonstrates the design and utility of a combined opto-dialysis probe, which allows measurement of local neurotransmitter levels during optical stimulation and the ability to locally apply pharmacological agents. The combined opto-dialysis system described here is likely to be useful in dissecting the role of specific neuronal populations and their interaction with their neighbors in the control of a wide variety of behaviors. Interestingly and importantly, we show that increased wakefulness produced by optical stimulation of BF cholinergic neurons requires cholinergic actions on neighboring non-cholinergic neurons. Thus, BF cholinergic neurons may affect behavioral state, not via direct actions on the cortical mantle but via activation of wake-promoting BF non-cholinergic neurons, in particular cortically projecting GABA/PV neurons.

\section{References}

Adamantidis AR, Zhang F, Aravanis AM, Deisseroth K, de Lecea L (2007) Neural substrates of awakening probed with optogenetic control of hypocretin neurons. Nature 450:420-424. CrossRef Medline

Adell A, Artigas F (2004) The somatodendritic release of dopamine in the ventral tegmental area and its regulation by afferent transmitter systems. Neurosci Biobehav Rev 28:415-431. CrossRef Medline

Anaclet C, Pedersen NP, Ferrari LL, Venner A, Bass CE, Arrigoni E, Fuller PM (2015) Basal forebrain control of wakefulness and cortical rhythms. Nat Commun 6:8744. CrossRef Medline

Aravanis AM, Wang LP, Zhang F, Meltzer LA, Mogri MZ, Schneider MB, Deisseroth K (2007) An optical neural interface: in vivo control of rodent motor cortex with integrated fiberoptic and optogenetic technology. J Neural Eng 4:S143-S156. CrossRef Medline

Brown RE, Basheer R, McKenna JT, Strecker RE, McCarley RW (2012) Control of sleep and wakefulness. Physiol Rev 92:1087-1187. CrossRef Medline

Cape EG, Manns ID, Alonso A, Beaudet A, Jones BE (2000) Neurotensininduced bursting of cholinergic basal forebrain neurons promotes gamma and theta cortical activity together with waking and paradoxical sleep. J Neurosci 20:8452-8461. Medline

Carter ME, Yizhar O, Chikahisa S, Nguyen H, Adamantidis A, Nishino S, Deisseroth K, de Lecea L (2010) Tuning arousal with optogenetic modulation of locus coeruleus neurons. Nat Neurosci 13:1526-1533. CrossRef Medline

Deisseroth K (2015) Optogenetics: 10 years of microbial opsins in neuroscience. Nat Neurosci 18:1213-1225. CrossRef Medline

Duque A, Balatoni B, Detari L, Zaborszky L (2000) EEG correlation of the discharge properties of identified neurons in the basal forebrain. J Neurophysiol 84:1627-1635. Medline

Duque A, Tepper JM, Detari L, Ascoli GA, Zaborszky L (2007) Morphological characterization of electrophysiologically and immunohistochemically identified basal forebrain cholinergic and neuropeptide Y-containing neurons. Brain Struct Funct 212:55-73. CrossRef Medline

Franklin KBJ, Paxinos G (2008) The mouse brain in stereotaxic coordinates, Ed 3. San Diego: Academic.

Freund TF, Meskenaite V (1992) gamma-Aminobutyric acid-containing basal forebrain neurons innervate inhibitory interneurons in the neocortex. Proc Natl Acad Sci U S A 89:738-742. CrossRef Medline

Fuller PM, Sherman D, Pedersen NP, Saper CB, Lu J (2011) Reassessment of the structural basis of the ascending arousal system. J Comp Neurol 519: 933-956. CrossRef Medline

Han Y, Shi YF, Xi W, Zhou R, Tan ZB, Wang H, Li XM, Chen Z, Feng G, Luo M, Huang ZL, Duan S, Yu YQ (2014) Selective activation of cholinergic basal forebrain neurons induces immediate sleep-wake transitions. Curr Biol 24:693-698. CrossRef Medline

Hassani OK, Lee MG, Henny P, Jones BE (2009) Discharge profiles of identified GABAergic in comparison to cholinergic and putative glutamatergic basal forebrain neurons across the sleep-wake cycle. J Neurosci 29: 11828-11840. CrossRef Medline

Henny P, Jones BE (2008) Projections from basal forebrain to prefrontal cortex comprise cholinergic, GABAergic and glutamatergic inputs to py- ramidal cells or interneurons. Eur J Neurosci 27:654-670. CrossRef Medline

Irmak SO, de Lecea L (2014) Basal forebrain cholinergic modulation of sleep transitions. Sleep 37:1941-1951. CrossRef Medline

Jones BE (2004) Activity, modulation and role of basal forebrain cholinergic neurons innervating the cerebral cortex. Prog Brain Res 145:157-169. CrossRef Medline

Kaur S, Junek A, Black MA, Semba K (2008) Effects of ibotenate and ${ }^{192} \mathrm{IgG}$ saporin lesions of the nucleus basalis magnocellularis/substantia innominata on spontaneous sleep and wake states and on recovery sleep after sleep deprivation in rats. J Neurosci 28:491-504. CrossRef Medline

Keski-Rahkonen P, Lehtonen M, Ihalainen J, Sarajärvi T, Auriola S (2007) Quantitative determination of acetylcholine in microdialysis samples using liquid chromatography/atmospheric pressure spray ionization mass spectrometry. Rapid Commun Mass Spectrom 21:2933-2943. CrossRef Medline

Kim T, Thankachan S, McKenna JT, McNally JM, Yang C, Choi JH, Chen L, Kocsis B, Deisseroth K, Strecker RE, Basheer R, Brown RE, McCarley RW (2015) Cortically projecting basal forebrain parvalbumin neurons regulate cortical gamma band oscillations. Proc Natl Acad Sci U S A 112: 3535-3540. CrossRef Medline

Kolisnyk B, Guzman MS, Raulic S, Fan J, Magalhães AC, Feng G, Gros R, Prado VF, Prado MA (2013) ChAT-ChR2-EYFP mice have enhanced motor endurance but show deficits in attention and several additional cognitive domains. J Neurosci 33:10427-10438. CrossRef Medline

Lee MG, Hassani OK, Alonso A, Jones BE (2005) Cholinergic basal forebrain neurons burst with theta during waking and paradoxical sleep. J Neurosci 25:4365-4369. CrossRef Medline

Lin SC, Gervasoni D, Nicolelis MA (2006) Fast modulation of prefrontal cortex activity by basal forebrain noncholinergic neuronal ensembles. J Neurophysiol 96:3209-3219. CrossRef Medline

Lin SC, Brown RE, Hussain Shuler MG, Petersen CC, Kepecs A (2015) Optogenetic dissection of the basal forebrain neuromodulatory control of cortical activation, plasticity, and cognition. J Neurosci 35:13896-13903. CrossRef Medline

Manns ID, Alonso A, Jones BE (2003) Rhythmically discharging basal forebrain units comprise cholinergic, GABAergic, and putative glutamatergic cells. J Neurophysiol 89:1057-1066. CrossRef Medline

Martinez-Murillo R, Villalba RM, Rodrigo J (1990) Immunocytochemical localization of cholinergic terminals in the region of the nucleus basalis magnocellularis of the rat: a correlated light and electron microscopic study. Neuroscience 36:361-376. CrossRef Medline

McKenna JT, Yang C, Franciosi S, Winston S, Abarr KK, Rigby MS, Yanagawa Y, McCarley RW, Brown RE (2013) Distribution and intrinsic membrane properties of basal forebrain GABAergic and parvalbumin neurons in the mouse. J Comp Neurol 521:1225-1250. CrossRef Medline

Metherate R, Cox CL, Ashe JH (1992) Cellular bases of neocortical activation: modulation of neural oscillations by the nucleus basalis and endogenous acetylcholine. J Neurosci 12:4701-4711. Medline

Porkka-Heiskanen T, Strecker RE, McCarley RW (2000) Brain sitespecificity of extracellular adenosine concentration changes duirng sleep deprivation and spontaneous sleep: an in vivo microdialysis study. Neuroscience 99:507-517. CrossRef Medline

Prokai L, Fryèák P, Stevens SM Jr, Nguyen V (2008) Measurement of acetylcholine in rat brain microdialysates by LC-isotope dilution tandem MS. Chromatographia 68:S101-S105. CrossRef Medline

Sarter M, Bruno JP (2000) Cortical cholinergic inputs mediating arousal, attentional processing and dreaming: differential afferent regulation of the basal forebrain by telencephalic and brainstem afferents. Neuroscience 95:933-952. CrossRef Medline

Semba K (2000) Multiple output pathways of the basal forebrain: organization, chemical heterogeneity, and roles in vigilance. Behav Brain Res 115: 117-141. CrossRef Medline

Shi YF, Han Y, Su YT, Yang JH, Yu YQ (2015) Silencing of cholinergic basal forebrain neurons using archaerhodopsin prolongs slow-wave sleep in mice. PLoS One 10:e130130. CrossRef Medline

Szymusiak R (1995) Magnocellular nuclei of the basal forebrain: substrates of sleep and arousal regulation. Sleep 18:478-500. Medline

Taylor H, Schmiedt JT, Carçak N, Onat F, Di Giovanni G, Lambert R, Leresche N, Crunelli V, David F (2014) Investigating local and long-range neuronal network dynamics by simultaneous optogenetics, reverse mi- 
crodialysis and silicon probe recordings in vivo. J Neurosci Methods 235: 83-91. CrossRef Medline

Vazquez J, Baghdoyan HA (2001) Basal forebrain acetylcholine release during REM sleep is significantly greater than during waking. Am J Physiol Regul Integr Comp Physiol 280:R598-R601. CrossRef Medline

Xu M, Chung S, Zhang S, Zhong P, Ma C, Chang WC, Weissbourd B, Sakai N, Luo L, Nishino S, Dan Y (2015) Basal forebrain circuit for sleep-wake control. Nat Neurosci 18:1641-1647. CrossRef Medline

Yang C, McKenna JT, Zant JC, Winston S, Basheer R, Brown RE (2014) Cholinergic neurons excite cortically projecting basal forebrain GABAergic neurons. J Neurosci 34:2832-2844. CrossRef Medline

Yang C, McKenna JT, Brown (2015) Basal forebrain vGluT2-positive neu- rons: electrophysiological properties and cholinergic modulation. Soc Neurosci Abstr 730.14.

Yizhar O, Fenno LE, Davidson TJ, Mogri M, Deisseroth K (2011) Optogenetics in neural systems. Neuron 71:9-34. CrossRef Medline

Zaborszky L, Duque A (2000) Local synaptic connections of basal forebrain neurons. Behav Brain Res 115:143-158. CrossRef Medline

Zaborszky L, Duque A (2003) Sleep-wake mechanisms and basal forebrain circuitry. Front Biosci 8:d1146-d1169. CrossRef Medline

Zhao S, Ting JT, Atallah HE, Qiu L, Tan J, Gloss B, Augustine GJ, Deisseroth K, Luo M, Graybiel AM, Feng G (2011) Cell type-specific channelrhodopsin-2 transgenic mice for optogenetic dissection of neural circuitry function. Nat Methods 8:745-752. CrossRef Medline 